\title{
Oral microbial pathogens isolates in newly diagnosed HIV positive patients. A baseline survey of the sociale institute of health and hygiene of Dakar.
}

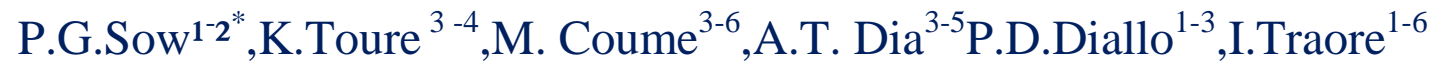 \\ ${ }^{1}$ Social institute of Health and hygiene of Dakar ${ }^{2}$ University of Bambey ${ }^{3}$ Cheikh AntaDiop University of Dakar \\ ${ }^{4}$ Hospital center of Fann ${ }^{5}$ Health Institute and development \\ ${ }^{6}$ Aristide le Dantec Hospital of Dakar-Bacteriology laboratory \\ Correspondence: Dr Papa gallo sow BP : 5856 Dakar- Fann /Sénègal
}

\begin{abstract}
The subjects were evaluated in correlation of the CD4 count, viral load and types of oral microbes isolated and the fungal infections in newly diagnosed patients with HIV infection prior to commencement of the highly active anti-retroviral therapy (HAART).

Fifty - seven newly diagnosed HIV positive patients were included in this on going study. The CD4 count, viral load were obtained. Oral examination was carried out and Buccal swabs was taken for Microscopy, Culture and Sensitivity. Males were $14(24.6 \%)$ and $43(75.4 \%)$ were females. About 39\% of the patients are within 30-39 years. The pattern of microbial pathogens isolated were: Pseudomonas spp 16.5\%, Klebsiella spp. 15.3\%, Proteus spp. $12.9 \%$ Staphylococcus aureus in $12.9 \%$ Escherichia coli $9.40 \%$, Candida albican $7.1 \%$, Streptococcus faecalis $2.4 \%$, Non Haemolytic streptococcus $2.4 \%$ and Staphylococcus albus 21.2\%. Concomitant tuberculosis infection was found in 3 patients .An inverse correlation was noted between the CD4 count and the viral load, which was statistically significant. The frequency of the oral lesions and the microbial isolates increased with high viral load. Seventy percent of the newly diagnosed HIV patients had CD4 counts less than $500 \mathrm{~mm}^{3}$.

The reduction of the oral microbial load may decrease the incidence of opportunistic infection. Unmet oral health needs of people living with HIV/AIDS have been consistently documented and finding new strategies for meeting these needs is urgent.
\end{abstract}

Keywords: Viral load, CD4 count, Oral, Microbial isolates. HIV infection.

\section{INTRODUCTION}

Human Immune Deficiency Virus (HIV) infection is one of the most devastating infections in modern times. An estimated 40.3 million HIV infections and 10 million AIDS cases have been reported worldwide. The brunt of the disease is largely borne by communities in the sub Saharan Africa where an estimated 28 million people are living with HIV/AIDS (UNAIDS/WHO,2010). Oral manifestations of HIV occur in approximately 30-80\% of all affected patients, and the factors which predispose to the expression of these lesions include; CD4 count $(<200 \mathrm{cells} / \mathrm{mm})$, viral load, xerostomia, poor oral hygiene and smoking (EEC,1993). Although the commonest oral lesion is the opportunistic fungal infection (candidiasis), 'normal' microbial flora or commensals of the oral mucosa which are locked in the saliva, dental plague, gingival crevice, tonsils and pharynx may become invasive or virulent as a result of weakened immune defenses (Patton,1994). With reduced CD4 count in HIV infection, granulocytopenia occurs. When the value of the granulocytes falls below 500 cells/ml, and in the presence of an attendant anatomical barrier damage that follows the viral infection, invasion of the bloodstream by microorganisms is facilitated with resultant sepsis and death (Greenspan,1997).The periodontal tissues in the mouth provide a potentially weak barrier through which bacteria and their toxins can enter the connective tissues and systemic circulation. Therefore maintaining a low microbial load within the mouth should be seen as an essential component of preventive treatment regime in HIV positive patients.

The aim of the study

The aim of this study is to correlate the CD4 count, viral load and types of oral microbes isolated in newly diagnosed patients with HIV infection prior to commencement of the highly active anti-retroviral therapy (HAART). 


\section{Patients and Method}

Fifty - seven newly diagnosed HIV positive patients were consecutively included in this on going study. The CD4 count and viral load were obtained. Oral examination was carried out on each patient by the principal investigator (VNO) using the standard 'examination set' under adequate lightening. Buccal swab was taken and cultured directly into $10 \%$ Blood agar, Chocolate agar and Macconkey agar, these plates were taken to Medical Microbiology Department for incubation for $24 \mathrm{hrs}$ at $37^{\circ} \mathrm{C}$ under a septic procedures that conforms with standard universal precautions .

\section{RESULTS}

The data obtained was analyzed using the SPSS version 12 . There were $24.6 \%$ males and $75.4 \%$ female patients in all. $38.6 \%$ patients were traders, $29.8 \%$ were artisans, $10.5 \%$ were civil servants and $5.3 \%$ were house wives. $75.4 \%$ patients (male and female) were married. The CD4 count was found to be < 200 in $24.6 \%, 200-499$ in $43.9 \%$ and $>500$ in $22.8 \%$. Only 43 of the cases had results of the viral load documented. An inverse correlation was noted between the CD4 count and the viral load, which was statistically significant. The most common oral lesion was Psuedomembranous candidiasis in 47.6\%, Xerostomia in $14.3 \%$., Gingivitis in $13 \%$ and melanotic hyperpigmentation in $11.9 \%$. The pattern of microbial pathogens isolated were: Pseudomonas spp 16.5\%, Klebsiella spp. 15.3\% , Proteus spp. 12.9\% Staphylococcus aureus in $12.9 \%$ Escherichia coli $9.40 \%$, Candida albican 7.1\%, Streptococcus faecalis $2.4 \%$, Non Haemolytic streptococcus $2.4 \%$ and Staphylococcus albus $21.2 \%$ (doubtful pathogens). The overall growth pattern showed $20 \%$ triple isolates, $40 \%$ multiple isolates, $30 \%$ showed mono isolates and $10 \%$ yielded no growth. The frequency of the oral lesions as well as the microbial isolates was found to increase with high viral load . In this study the most common systemic disease associated with HIV infection was pulmonary tuberculosis, which was found in 3 cases.

\section{DISCUSSION}

To date, 300 different species of micro organisms are known to be associated with the oral cavity but only half of the bacteria can be cultured. In health, the normal oral ecosystem is relatively stable in spite of its complexity (Duerden et al., 1995) but many endogenous and exogenous factors may affect the composition and metabolic activities of the oral microflora. The CD4 counts and viral loads are standard tools for monitoring the response to treatment and they are markers of disease severity and progression in patients with HIV infection and AIDs. The principal cellular target of HIV infection is the CD4 T-helper cells and the depletion of these cells is a central factor in the progression of HIV infection to AIDS as a disease. This cellular immune dysfunction results in the inability of monocytes and macrophages to kill intracellular pathogens effectively (Amsterdam ,1998) so that various opportunistic pathogens can produce infection in such instances. In Senegal, the CD4 cell count in healthy individuals has been found to range from $636 / \mathrm{mm}^{3}$ to $977 / \mathrm{mm}^{3}$. However, in developed countries, the mean value of CD4 cell count in normal individuals ranges from $1000 / \mathrm{mm}^{3}$ to $1100 / \mathrm{mm}^{3}$. The relatively lower values in Senegal and probably in other Africa countries may be due to the constant exposure of inhabitants in these countries to a large number of pathogens. (Marsh et al.,1992) When the presumably 'normal' oral microorganism become invasive, they can establish infections at other sites remote from the mouth (e.g. infective endocarditis) and they may also spread directly along tissue planes (e.g. cerebral abscess). Therefore, the delicate balance between the resident commensal or 'normal' oral flora and the host must be maintained in healthy humans. (Olaleye et al.,2006)

In this study, $70 \%$ of the newly diagnosed HIV patients had CD4 counts less than $500 \mathrm{~mm}^{3}$ and a statistically significant inverse correlation was observed between the CD4 count and viral load. The isolation of invasive and pathogenic microbes such as Pseudomonas, Escherichia Coli, Klebsiella spp, Streptococcus faecalis and Staphylococcus aureus ( some of which are predominantly colonic commensals and transitional bacteria) from the oral cavity may suggest the presence of depressed immune status. Moreover, some patients in the bid to get well, usually visit the herbalist and spiritual houses for various concoctions which are usually prepared in unhygienic situations The growth pattern that showed triple and double isolate also reinforced that the patients were immune depressed. In Senegal the CD4 cell count in healthy individuals has been found to range from 636 to 977/mm3 (Senegal Epidemiological fact sheet ,2004). However, in developed countries, the mean value of CD4 cell count in normal individuals range from 1000 to $1100 / \mathrm{mm} 3$. The relatively lower values in Nigeria and probably in other African countries may be due to the constant exposure of inhabitants in these countries to a large number of pathogens. (Olaleye et al., 2006) 


\section{CONCLUSION}

Oral health needs of people living with HIV/AIDs have been consistently documented and finding new strategies for meeting these needs is urgent. Therefore the desire for prophylactic oral hygiene measures to reduce and if possible eliminate these invasive microbial isolates and the incorporation of such in the holistic management of the HIV infected patients cannot be compromised.

\section{REFERENCES}

[1]. Amsterdam (1998), Infections associated with immunodeficiency and immunosuppression. Topley $\&$ Wilson's Microbiology and microbial infections Volume 3. pp $9^{\text {th }}$ edition.

[2]. Duerden BI, Wade WG et al (eds) (1995) Medical and Dental Aspects of Anaerobes Science Reviews, Northwood, 1-85.

[3]. EEC- Clearinghouse on Oral problems in HIV infection and WHO collaborating Center on Oral manifestations of the immunodeficiency virus: An update of the classification and diagnostic criteria of oral lesions in HIV infections. J Oral Pathol Med. 1993; 22: 289-291.

[4]. Greenspan JS(1997) Sentinels and signposts: The epidemiology and significance of the oral manifestation of HIV disease. Oral Dis. (Suppl 1): S13-S17.

[5]. Marsh PD, Martin MV, (1992) Oral microbiology, $3^{\text {rd }}$ edn Chapman and Hall. London

[6]. Olaleye D.O, Tekena O Harry, Georgina N Odiabo(2006).The Virology and dynamics of the epidemic AIDS in Nigeria; A nation on the threshold, pp 46.

[7]. Oral Health Care for people with HIV infections. HIV Clinical Guidelines for the Primary care practitioner; New York State Department of Health AIDS Institute. December 2001

[8]. Patton LL(1994) Sensitivity, specificity and predictive value of oral opportunistic infection in adults with HIV-related disease as markers for immune suppression and AIDS. Oral Surg. Oral Pathol. 77 (4): 344-349

[9]. Senegal Epidemiological fact sheet $N^{\circ} 11$, September 2004 UNAIDS/WHO Report on the global AIDS Epidemic, May 2010 